\title{
Postoperative complications: an observational study of trends in the United States from 2012 to 2018
}

\author{
Emilie Even Dencker ${ }^{1,2}$, Alexander Bonde ${ }^{1,2}$, Anders Troelsen ${ }^{3,5}$, Kartik Mangudi Varadarajan $^{4}$ and \\ Martin Sillesen $1,2,5^{*}$
}

\begin{abstract}
Background: Postoperative complications continue to constitute a major issue for both the healthcare system and the individual patient and are associated with inferior outcomes and higher healthcare costs. The objective of this study was to evaluate the trends of postoperative complication rates over a 7-year period.

Methods: The NSQIP datasets from 2012 to 2018 were used to assess 30-day complication incidence rates including mortality rate following surgical procedures within ten surgical subspecialties. Multivariable logistic regression was used to associate complication rates with dataset year, while adjusting for relevant confounders.

Results: A total of 5,880,829 patients undergoing major surgery were included. Particularly the incidence rates of four complications were found to be decreasing: superficial SSI (1.9 to 1.3\%), deep SSI (0.6 to $0.4 \%)$, urinary tract infection (1.6 to $1.2 \%$ ) and patient unplanned return to the operating room (3.1 to $2.7 \%$ ). Incidence rate for organ/space SSI exhibited an increase (1.1 to 1.5\%). When adjusted, regression analyses indicated decreased odds ratios (OR) through the study period years for particularly deep SSI OR 0.92 [0.92-0.93], superficial SSI OR 0.94 [0.94-0.94] and acute renal failure OR 0.96 [0.95-0.96] as the predictor variable (study year) increased $(p<0.01)$. However, OR's for organ/space SSI 1.05 [1.05-1.06], myocardial infarction 1.01 [1.01-1.02] and sepsis 1.01 [1.01-1.02] increased slightly over time (all $\mathrm{p}<0.01)$.
\end{abstract}

Conclusions: Incidence rates for the complications exhibited a stable trend over the study period, with minor in or decreases observed.

Keywords: Surgery, Complications, Trends

\section{Background}

The total volume of major surgeries performed annually worldwide was estimated to be 312.9 million in 2012-an increase of $38.2 \%$ from 2004 [1]. Studies estimate that $7-15 \%$ of patients undergoing major surgery will experience a postoperative complication (PC) $[2,3]$.

\footnotetext{
*Correspondence: Martin.Sillesen@regionh.dk

1 Department of Surgical Gastroenterology and Transplantation C-TX,

Copenhagen University Hospital, Blegdamsvej 9, Copenhagen $\varnothing$,

2100 Rigshospitalet, Denmark

Full list of author information is available at the end of the article
}

Furthermore, the overall postoperative mortality rate is reported to vary from 0.79 to $5.7 \%$ [4]. According to the Clavien-Dindo classification, a PC is defined as any deviation from the normal postoperative course, meaning that the severity ranges from non-life-threatening complications with no lasting disability to fatal outcomes [5, 6]. In the individual level, PCs can have major impact on the individual patient, potentially resulting in a decline of both quality of life as well as functional performance [4]. From a societal standpoint, PCs account for a large financial burden in the form of additional health care expenses 
when a patient requires Intensive Care Unit (ICU) treatment, reoperation or readmission, thereby increasing healthcare costs $[5,7,8]$ in an estimated order of magnitude between $\$ 11,626$ and $\$ 19,626$ in additional healthcare expenses per patient $[7,9]$.

A number of studies have highlighted the scope of the problem within specific surgical subspecialties. PC trends following total knee arthroplasty (TKA) has been reported to decrease during a 10-year study period (2006-2016) [10]. However, studies assessing this issue in general across multiple subspecialties and thereby estimating the overall extent of postoperative complications and its trend over time are lacking. The primary objective in this study was to evaluate the trends for postoperative complication rates over a 7-year period (2012-2018). Our secondary objective was to assess whether complications incidences exhibited significant changes over the study period.

We hypothesized that the rates of incidence for fourteen specified postoperative complications would exhibit minor changes over this 7-year period.

\section{Methods}

This study utilized the American College of Surgeons (ACS) National Surgical Quality Improvement Program (NSQIP). NSQIP is a national database containing data on multiple surgical outcomes from more than 680 participating hospital sites in the United States and data in the NSQIP database are captured by a site's trained and certified Surgical Clinical Reviewer (SCR) [11]. The study was approved by the ACS and the Massachusetts
General Hospital Institutional Review Board. Findings are reported in accordance with STROBE guidelines.

\section{Patient selection}

We included patients admitted from the period January 1st, 2012 to December 31st, 2018. All patients were $>18$ years. Patients with subspecialty listed as "Other", "Unknown" or "Interventional radiology" were excluded from the study (Fig. 1). In the NSQIP data, maximum age was coded as $90+$. This was recoded as 90 years of age in our calculations to enable calculation of median age. Elective as well as emergency cases were included. Preoperative variables were collected including demographics, comorbidities and surgical subspecialty. We identified patients with any of the following clinical outcomes occurring within 30 days of the index procedure: superficial surgical site infection (SSI), deep incisional SSI, organ/space SSI, pneumonia, pulmonary embolism, acute renal failure, urinary tract infection, Cerebrovascular Accident (CVA)/stroke with neurological deficit, cardiac arrest requiring cardiopulmonary resuscitation (CPR), Myocardial Infarction (MI), deep venous thrombosis (DVT), sepsis/septic shock, and unplanned return to the operating room (OR). All cases of 30-day postoperative all-cause mortality were collected as well. Outcomes were defined by NSQIP and registered by the NSQIP SCRs [12].

\section{Statistical analysis}

Descriptive statistics were performed to describe demographics, postoperative morbidity and mortality from

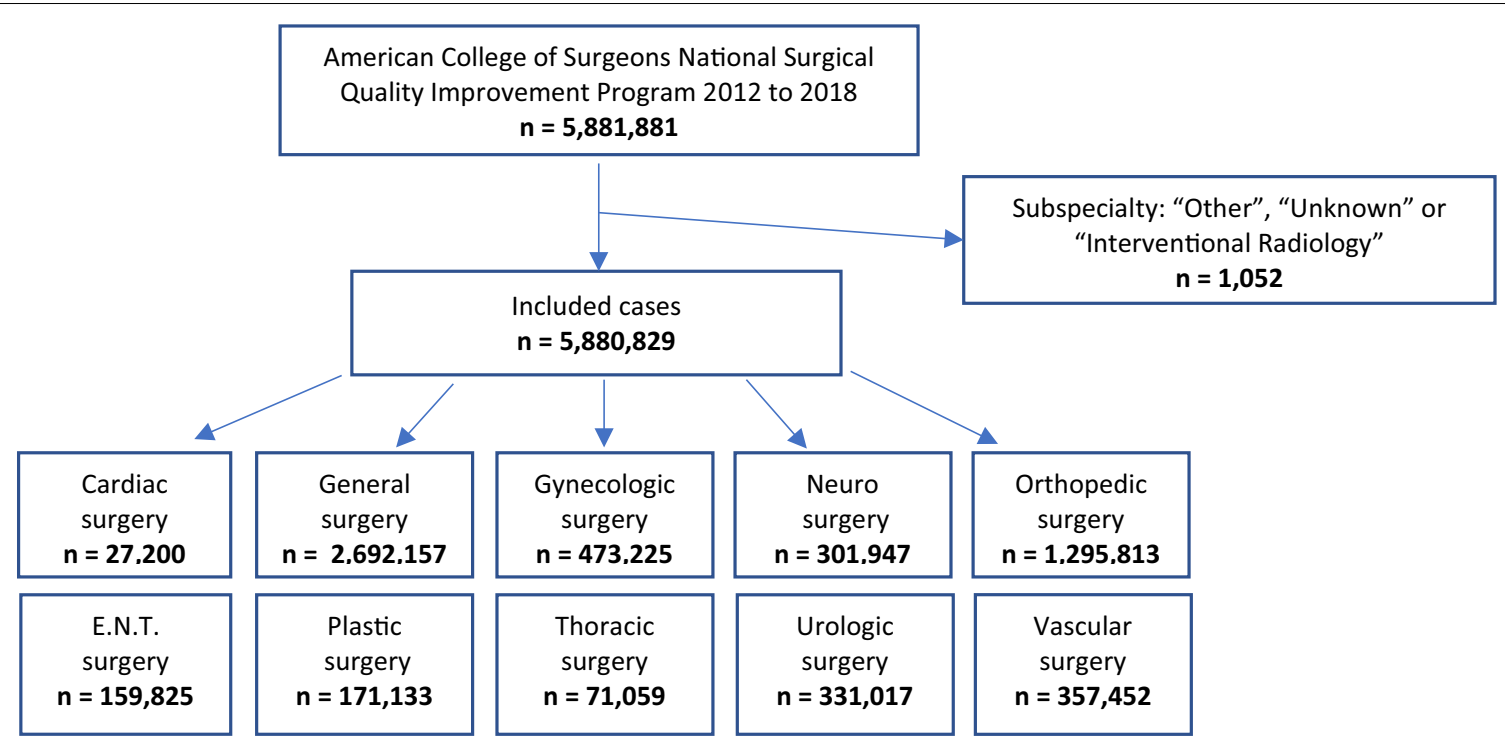

Fig. 1 Flowchart of patient cases from American College of Surgeons (ACS) National Surgical Quality Improvement Program (NSQIP) included in the study. All cases with available information on sex, age and surgical subspecialty were included 
2012 to 2018. We calculated postoperative complication incidence rates over the study period for each outcome within the various subspecialties. Data are presented as medians with interquartile range, or percentages for dichotomous variables where appropriate. A logistic regression model was used to associate complication incidences with study year, the latter modelled as a continuous variable. This approach was chosen in order to allow for an assessment of whether the slope of the incidence curve in or decreased over the study years when adjusting for relevant confounders. Data are presented as odds ratios (OR) with [95\% confidence intervals (CI)] for each postoperative complication. Analyses results are presented as univariable as well as multivariable regression, adjusted for the variables: surgical subspecialty, age, gender, body mass index (BMI), American Society of Anesthesiology (ASA) score, diabetes (including both insulin and non-insulin requiring), chronic obstructive pulmonary disease (COPD), blood transfusion, smoking, preoperative renal failure and hypertension requiring medication.

The choice of these covariates was based on the desire to include covariates with a reasonably documented association with PCs and thus model overall patient frailty. We opted to use this approach as opposed to a data driven approach (e.g., stepwise regression), as studies have indicated a frequent over-inclusion of covariates in disagreement with expert consensus using this approach [13].

BMI was calculated using the formula $\mathrm{BMI}=$ weight (lb)/[height (in) $] 2 \times 703$.

All statistical analysis was performed in $\mathrm{R}$ version 3.5.2 [14]. Regression modelling was made using the standard $\mathrm{R}$ glm approach. A p-value $<0.05$ was considered statistically significant.

\section{Missing data}

Missing data was assumed to be missing at random and was handled by listwise exclusion in the multivariable modelling. An overview of missing data is presented in Additional file 1: Table S1 and comprised of $<0.001 \%$ of the total cohort. As such, missing data was not considered to have relevant impact on the dataset, and imputation was thus not done.

\section{Results}

A total of 5,880,820 patients who underwent major surgery from 2012 to 2018 were included in the study (Table 1 and Fig. 1). The most common of the included comorbidities (Table 1) was hypertension (44.9\%), followed by diabetes (15.5\%) and Chronic Obstructive Pulmonary Disease (COPD, 4.4\%). Furthermore, 5.4\% of patients experienced sepsis preoperatively. General surgery accounted for the majority of the surgical procedures with a total of 2,692,157 (45.8\%) performed procedures followed by orthopedic surgery (22.0\%) (Table 1 and Fig. 1). Median ASA scores were 2 for all included study years.

Postoperative complication incidences and rates for all surgical procedures combined are shown in Table 2 . From 2012 to 2018 the incidence rates for the following complications appear decreasing: superficial SSI (1.9 to $1.3 \%)$, deep SSI (0.6 to $0.4 \%)$, urinary tract infection (1.6 to $1.2 \%$ ) and patient unplanned return to the operating room (3.1 to $2.7 \%$ ). The incidence rate for postoperative organ/space SSI for all surgical procedures combined exhibit an increase (1.1 to $1.5 \%)$. We observed no relevant changes in the nine remaining studied PC incidence rates within the study period (Table 2 and Fig. 2).

Overall, similar trends were generally found when stratified into surgical subspecialties and are presented in Additional file 1: Table S2). Figure 3 illustrates the trends for all studied complications within the two largest subspecialties: general and orthopedic surgery.

Results of both the univariable and multivariable regression model are show in Table 3. When corrected for relevant confounders, regression analyses indicated decreased odds ratios (OR) for the majority of the studied complications-most apparent for deep SSI OR 0.92 [0.92-0.93], superficial SSI OR 0.94 [0.94-0.94] and acute renal failure OR 0.96 [0.95-0.96], as the predictor variable (study year) increased $(\mathrm{p}<0.01)$. In contrast, OR for organ/space SSI, MI and sepsis increased over time (Table 3).

Odds ratios for the remaining studied complications were all statistically significant with $\mathrm{p}$-values $<0.05$ but exhibited minor decreases or increases in OR (Table 3).

\section{Discussion}

In this study we investigated the development of complication incidences following surgical procedures in the US as registered in the NSQIP database from 2012 to 2018. We found that several complication incidences exhibited a temporal decline during the study period, in which superficial SSI, deep SSI, urinary tract infection and unplanned return to operating room appear to have the most declining rates. Meanwhile the incidence rate for organ/space SSI increased (Table 2). These findings were largely consistent throughout various subspecialties (Additional file 1: Table S2).

In 2014 more than 14 million operating room procedures were performed during inpatient hospital stays in the US, as reported by the Agency for Healthcare Research and Quality (AHRQ) [15]. In addition, AHRQ reported 17.3 million outpatient surgeries in 2012[16]. The volume of surgical procedures performed annually 
Table 1 Demographics of the 5,880,829 surgical cases from ACS NSQIP from 2012-2018. Values are median [Interquartile Range] or number/proportion

\begin{tabular}{|c|c|c|c|c|c|c|c|c|}
\hline $\begin{array}{l}\text { Procedure } \\
\text { year }\end{array}$ & 2012 & 2013 & 2014 & 2015 & 2016 & 2017 & 2018 & All \\
\hline Individuals & 543,815 & 651,770 & 750,792 & 885,350 & $1,000,195$ & $1,028,574$ & $1,020,333$ & $5,880,829$ \\
\hline Age; years & 58 [45-69] & 58 [45-69] & 58 [44-69] & 58 [45-69] & 58 [45-69] & 58 [45-69] & $59[45-70]$ & 58 [45-69] \\
\hline Sex; male & $\begin{array}{l}232,164 \\
(42.7 \%)\end{array}$ & $\begin{array}{l}276,715 \\
(42.5 \%)\end{array}$ & $\begin{array}{l}324,125 \\
(43.2 \%)\end{array}$ & $\begin{array}{l}384,005 \\
(43.4 \%)\end{array}$ & $\begin{array}{l}434,783 \\
(43.5 \%)\end{array}$ & $\begin{array}{l}443,156 \\
(43.1 \%)\end{array}$ & $\begin{array}{l}437,897 \\
(42.9 \%)\end{array}$ & $\begin{array}{l}2,532,845 \\
(43.1 \%)\end{array}$ \\
\hline $\mathrm{BMI} ; \mathrm{kg} / \mathrm{m}^{2}$ & $\begin{array}{l}28.3 \\
{[24.4-33.5]}\end{array}$ & $\begin{array}{l}28.4 \\
{[24.4-33.7]}\end{array}$ & $\begin{array}{l}28.7[24.5- \\
33.8]\end{array}$ & $\begin{array}{l}28.7[24.7- \\
33.9]\end{array}$ & $\begin{array}{l}28.9[24.8- \\
34.0]\end{array}$ & $\begin{array}{l}28.9[24.9- \\
34.1]\end{array}$ & $\begin{array}{l}28.9[24.9- \\
34.0]\end{array}$ & 28.6 [24.8-33.9] \\
\hline $\begin{array}{l}\text { Surgery dura- } \\
\text { tion }\end{array}$ & $\begin{array}{l}86 \min \\
{[50-144]}\end{array}$ & $\begin{array}{l}86 \min \\
{[50-143]}\end{array}$ & $\begin{array}{l}86 \min \\
{[50-142]}\end{array}$ & $\begin{array}{l}84 \min \\
{[50-139]}\end{array}$ & $\begin{array}{l}84 \min \\
{[50-138]}\end{array}$ & $\begin{array}{l}85 \min \\
{[51-139]}\end{array}$ & $\begin{array}{l}85 \min \\
(51-141)\end{array}$ & $85 \min [50-140]$ \\
\hline \multicolumn{9}{|l|}{ Ethnicity: } \\
\hline $\begin{array}{l}\text { American } \\
\text { Indian/ Alaska } \\
\text { Native }\end{array}$ & $2,639(0.5 \%)$ & 4,205 (0.6\%) & $5,390(0.7 \%)$ & $5,563(0.6 \%)$ & $5,408(0.5 \%)$ & 5,109 (0.5\%) & $5,145(0.5 \%)$ & $33,459(0.6 \%)$ \\
\hline Asian & $16,423(3.0 \%)$ & 18,845 (2.9\%) & $21,080(2.8 \%)$ & 24,002 (2.7\%) & $26,504(2.6 \%)$ & $29,518(2.9 \%)$ & $30,423(3.0 \%)$ & $\begin{array}{l}166,795 \\
(2.8 \%)\end{array}$ \\
\hline $\begin{array}{l}\text { Black or Afri- } \\
\text { can American }\end{array}$ & $50,557(9.3 \%)$ & 62,767 (9.6\%) & 75,877 (10.1\%) & $88,936(10.0 \%)$ & $\begin{array}{l}100,507 \\
(10.0 \%)\end{array}$ & $\begin{array}{l}105,298 \\
(10.2 \%)\end{array}$ & $\begin{array}{l}102,018 \\
(10.0 \%)\end{array}$ & $\begin{array}{l}585,960 \\
(10.0 \%)\end{array}$ \\
\hline Hispanic & $5,688(1.0 \%)$ & $8,436(1.3 \%)$ & $11,258(1.5 \%)$ & $13,635(1.5 \%)$ & $17,953(1.8 \%)$ & $22,131(2.2 \%)$ & $24,678(2.4 \%)$ & $\begin{array}{l}103,779 \\
(1.8 \%)\end{array}$ \\
\hline $\begin{array}{l}\text { Native Hawai- } \\
\text { ian/ Pacific } \\
\text { Islander }\end{array}$ & $2,336(0.4 \%)$ & $2.722(0.4 \%)$ & $3,103(0.4 \%)$ & $3,469(0.4 \%)$ & $3,768(0.4 \%)$ & $3,762(0.4 \%)$ & $3,616(0.4 \%)$ & $22,776(0.4 \%)$ \\
\hline White & $\begin{array}{l}403,719 \\
(74.2 \%)\end{array}$ & $\begin{array}{l}483,949 \\
(74.3 \%)\end{array}$ & $\begin{array}{l}551,803 \\
(73.5 \%)\end{array}$ & $\begin{array}{l}640,518 \\
(72.3 \%)\end{array}$ & $\begin{array}{l}705,368 \\
(70.5 \%)\end{array}$ & $\begin{array}{l}722,782 \\
(70.3 \%)\end{array}$ & $\begin{array}{l}700,901 \\
(68.7 \%)\end{array}$ & $\begin{array}{l}4,209,040 \\
(71.6 \%)\end{array}$ \\
\hline $\begin{array}{l}\text { Unknown/ } \\
\text { Not reported }\end{array}$ & $62,453(11.5 \%)$ & 70,844 (10.9\%) & $82,281(11.0 \%)$ & $\begin{array}{l}109,227 \\
(12.3 \%)\end{array}$ & $\begin{array}{l}140,687 \\
(14.1 \%)\end{array}$ & $\begin{array}{l}139,974 \\
(13.6 \%)\end{array}$ & $\begin{array}{l}153,552 \\
(15.0 \%)\end{array}$ & $\begin{array}{l}759,018 \\
12.9 \%\end{array}$ \\
\hline \multicolumn{9}{|l|}{ Comorbidities } \\
\hline $\begin{array}{l}\text { Hyperten- } \\
\text { sion requiring } \\
\text { medication }\end{array}$ & $\begin{array}{l}249,610 \\
(45.9 \%)\end{array}$ & $\begin{array}{l}298,722 \\
(45.8 \%)\end{array}$ & $\begin{array}{l}337,796 \\
(45.0 \%)\end{array}$ & $\begin{array}{l}398,197 \\
(45.0 \%)\end{array}$ & $\begin{array}{l}447,427 \\
(44.7 \%)\end{array}$ & $\begin{array}{l}458,267 \\
(44.5 \%)\end{array}$ & $\begin{array}{l}448,949 \\
(44.0 \%)\end{array}$ & $\begin{array}{l}2,638,968 \\
(44.9 \%)\end{array}$ \\
\hline Diabetes & $82,348(15.1 \%)$ & 99,985 (15.3\%) & $\begin{array}{l}114,839 \\
(15.3 \%)\end{array}$ & $\begin{array}{l}136,865 \\
(15.5 \%)\end{array}$ & $\begin{array}{l}156,468 \\
(15.6 \%)\end{array}$ & $\begin{array}{l}160,816 \\
(15.6 \%)\end{array}$ & $\begin{array}{l}157,897 \\
(15.5 \%)\end{array}$ & $\begin{array}{l}909,218 \\
(15.5 \%)\end{array}$ \\
\hline COPD & $25,054(4.6 \%)$ & $30,229(4.6 \%)$ & $34,304(4.6 \%)$ & $40,980(4.6 \%)$ & $43,711(4.4 \%)$ & $43,623(4.2 \%)$ & $42,115(4.1 \%)$ & $\begin{array}{l}260,016 \\
(4.4 \%)\end{array}$ \\
\hline Ascites & 2,495 (0.5\%) & $2,616(0.4 \%)$ & $2,832(0.4 \%)$ & $3,182(0.4 \%)$ & $3,016(0.3 \%)$ & $2,991(0.3 \%)$ & $2,896(0.3 \%)$ & $20,028(0.3 \%)$ \\
\hline $\begin{array}{l}\text { Congestive } \\
\text { heart failure }\end{array}$ & 4,119 (0.8\%) & $5,421(0.8 \%)$ & $6,626(0.9 \%)$ & 8,221 (0.9\%) & 8,800 (0.9\%) & 8,538 (0.8\%) & 8,548 (0.8\%) & $50,273(0.9 \%)$ \\
\hline Dialysis & $\begin{array}{l}7,631 \\
(1.4 \%)\end{array}$ & $\begin{array}{l}9,062 \\
(1.4 \%)\end{array}$ & $\begin{array}{l}10,101 \\
(1.3 \%)\end{array}$ & 11,985 (1.4\%) & 13,070 (1.3\%) & $13,206(1.3 \%)$ & $13,049(1.3 \%)$ & $\begin{array}{l}78,104 \\
(1.3 \%)\end{array}$ \\
\hline $\begin{array}{l}\text { Disseminated } \\
\text { cancer }\end{array}$ & 11,287 (2.1\%) & $14,846(2.3 \%)$ & $17,846(2.3 \%)$ & $20,472(2.3 \%)$ & 22,355 (2.2\%) & $23,566(2.3 \%)$ & $23,252(2.3 \%)$ & $\begin{array}{l}133,624 \\
(2.3 \%)\end{array}$ \\
\hline Sepsis & 29,103 (5.4\%) & $33,796(5.2 \%)$ & $39,512(5.3 \%)$ & $47,468(5.4 \%)$ & $54,688(5.5 \%)$ & $57,643(5.6 \%)$ & 57,067 (5.6\%) & $\begin{array}{l}319,277 \\
(5.4 \%)\end{array}$ \\
\hline \multicolumn{9}{|c|}{ Surgical subspecialty: } \\
\hline Cardiac & $\begin{array}{l}4,028 \\
(0.7 \%)\end{array}$ & $\begin{array}{l}3,093 \\
(0.5 \%)\end{array}$ & $\begin{array}{l}3,693 \\
(0.5 \%)\end{array}$ & $\begin{array}{l}4,033 \\
(0.5 \%)\end{array}$ & $\begin{array}{l}4,281 \\
(0.4 \%)\end{array}$ & $\begin{array}{l}4,012 \\
(0.4 \%)\end{array}$ & $\begin{array}{l}4,060 \\
(0.4 \%)\end{array}$ & $\begin{array}{l}27,200 \\
(0.5 \%)\end{array}$ \\
\hline $\begin{array}{l}\text { General } \\
\text { surgery }\end{array}$ & $\begin{array}{l}277,926 \\
(51.1 \%)\end{array}$ & $\begin{array}{l}322,058 \\
(49.4 \%)\end{array}$ & $\begin{array}{l}360,397 \\
(48.0 \%)\end{array}$ & $\begin{array}{l}409,231 \\
(46.2 \%)\end{array}$ & $\begin{array}{l}445,639 \\
(44.5 \%)\end{array}$ & $\begin{array}{l}444,203 \\
(43.2 \%)\end{array}$ & $\begin{array}{l}432,703 \\
(42.4 \%)\end{array}$ & $\begin{array}{l}2,692,157 \\
(45.8 \%)\end{array}$ \\
\hline Gynecology & $\begin{array}{l}36,941 \\
(6.8 \%)\end{array}$ & $46,645(7.2 \%)$ & $55,339(7.4 \%)$ & $65,653(7.4 \%)$ & 77,744 (7.8\%) & $92,077(9.0 \%)$ & $98,826(9.7 \%)$ & $\begin{array}{l}473,225 \\
(8.0 \%)\end{array}$ \\
\hline Neurosurgery & $\begin{array}{l}23,585 \\
(4.3 \%)\end{array}$ & $32,640(5.0 \%)$ & $37,442(5.0 \%)$ & $46,665(5.3 \%)$ & $53,127(5.3 \%)$ & $54,856(5.3 \%)$ & $53,632(5.3 \%)$ & $\begin{array}{l}301,947 \\
(5.1 \%)\end{array}$ \\
\hline Orthopedics & $\begin{array}{l}92,592 \\
(17.0 \%)\end{array}$ & $\begin{array}{l}122,036 \\
(18.7 \%)\end{array}$ & $\begin{array}{l}153,320 \\
(20.4 \%) \\
\end{array}$ & $\begin{array}{l}197,868 \\
(22.3 \%) \\
\end{array}$ & $\begin{array}{l}235,029 \\
(23.5 \%) \\
\end{array}$ & $\begin{array}{l}243,991 \\
(23.7 \%) \\
\end{array}$ & $\begin{array}{l}250,977 \\
(24.6 \%)\end{array}$ & $\begin{array}{l}1,295,813 \\
(22.0 \%)\end{array}$ \\
\hline
\end{tabular}


Table 1 (continued)

\begin{tabular}{|c|c|c|c|c|c|c|c|c|}
\hline $\begin{array}{l}\text { Procedure } \\
\text { year }\end{array}$ & 2012 & 2013 & 2014 & 2015 & 2016 & 2017 & 2018 & All \\
\hline $\begin{array}{l}\text { Otolaryngol- } \\
\text { ogy } \\
\text { (ENT) }\end{array}$ & $\begin{array}{l}14,621 \\
(2.7 \%)\end{array}$ & 17,001 (2.6\%) & $20,859(2.8 \%)$ & $24,658(2.8 \%)$ & $28,147(2.8 \%)$ & $27,891(2.7 \%)$ & $26,648(2.6 \%)$ & $\begin{array}{l}159,825 \\
(2.7 \%)\end{array}$ \\
\hline Plastics & $\begin{array}{l}14,777 \\
(2.7 \%)\end{array}$ & $18,440(2.8 \%)$ & $21,108(2.8 \%)$ & $24,596(2.8 \%)$ & 29,382 (2.9\%) & 31,124 (3.0\%) & 31,706 (3.1\%) & $\begin{array}{l}171,133 \\
(2.9 \%)\end{array}$ \\
\hline Thoracic & $\begin{array}{l}7397 \\
(1.4 \%)\end{array}$ & 8375 (1.3\%) & 9420 (1.3\%) & 9788 (1.1\%) & $11,323(1.1 \%)$ & $12,298(1.2 \%)$ & $12,458(1.2 \%)$ & $\begin{array}{l}71,059 \\
(1.2 \%)\end{array}$ \\
\hline Urology & $\begin{array}{l}29,044 \\
(5.3 \%)\end{array}$ & $34,015(5.2 \%)$ & $39,632(5.3 \%)$ & $49,184(5.6 \%)$ & $57,963(5.8 \%)$ & $61,140(5.9 \%)$ & $60,039(5.9 \%)$ & $\begin{array}{l}331,017 \\
(5.6 \%)\end{array}$ \\
\hline Vascular & $\begin{array}{l}42,904 \\
(7.9 \%)\end{array}$ & 47,467 (7.3\%) & $49,582(6.6 \%)$ & $53,674(6.1 \%)$ & $57,560(5.8 \%)$ & $56,981(5.5 \%)$ & $49,284(4.8 \%)$ & $\begin{array}{l}357,452 \\
(6.1 \%)\end{array}$ \\
\hline
\end{tabular}

COPD chronic obstructive pulmonary disease, ENT ear-nose-throat, BMI body mass index

Table 2 Incidence rates for the 14 studied postoperative complications for all surgical procedures combined

\begin{tabular}{|c|c|c|c|c|c|c|c|c|}
\hline $\begin{array}{l}\text { Procedure } \\
\text { year }\end{array}$ & 2012 & 2013 & 2014 & 2015 & 2016 & 2017 & 2018 & All \\
\hline \multicolumn{9}{|c|}{ All surgical procedures: } \\
\hline $\begin{array}{l}\text { Unplanned } \\
\text { return to } \\
\text { OR: }\end{array}$ & $\mathbf{3 . 1 \%}(16,983)$ & $\mathbf{3 . 0 \%}(19,730)$ & $\mathbf{2 . 9} \%(21,822)$ & $\mathbf{2 . 9} \%(25,875)$ & $\mathbf{2 . 8} \%(27,548)$ & $\mathbf{2 . 7 \%}(27,916)$ & $\mathbf{2 . 7 \%}(27,260)$ & $\mathbf{2 . 8 \%}(167,134)$ \\
\hline Sepsis: & $1.4 \%(7,750)$ & $\mathbf{1 . 6 \%}(10,169)$ & $\mathbf{1 . 8 \%}(13,305)$ & $1.6 \%(14,593)$ & $1.6 \%(16,366)$ & $1.7 \%(17,250)$ & $1.6 \%(16,296)$ & $1.6 \%(95,729)$ \\
\hline $\begin{array}{l}\text { Superficial } \\
\text { SSI: }\end{array}$ & $\mathbf{1 . 9 \%}(10,062)$ & $\mathbf{1 . 7 \%}(11,065)$ & $\mathbf{1 . 6 \%}(12,195)$ & $\mathbf{1 . 5 \%}(13,072)$ & $1.4 \%(14,077)$ & $1.3 \%(13,713)$ & $1.3 \%(13,269)$ & $1.5 \%(87,453)$ \\
\hline $\begin{array}{l}\text { Organ/ } \\
\text { Space SSI: }\end{array}$ & $\mathbf{1 . 1} \%(5,775)$ & $\mathbf{1 . 2} \%(7542)$ & $1.2 \%(9305)$ & $\mathbf{1 . 3} \%(11,146)$ & $\mathbf{1 . 4 \%}(13,578)$ & $1.4 \%(14,664)$ & $\mathbf{1 . 5 \%}(14,986)$ & $1.3 \%(76,996)$ \\
\hline $\begin{array}{l}\text { Urinary tract } \\
\text { infection: }\end{array}$ & $1.6 \%(8,466)$ & $\mathbf{1 . 4 \%}(9162)$ & $1.3 \%(9,96)$ & $1.3 \%(11,606)$ & $\mathbf{1 . 2 \%}(12,166)$ & $\mathbf{1 . 2} \%(12,227)$ & $\mathbf{1 . 2} \%(12,345)$ & $1.3 \%(75,968)$ \\
\hline Pneumonia: & $\mathbf{1 . 1} \%(6,195)$ & $\mathbf{1 . 2 \%}(8052)$ & $1.3 \%(9732)$ & $\mathbf{1 . 3} \%(11,310)$ & $1.2 \%(11,831)$ & $\mathbf{1 . 1} \%(11,449)$ & $\mathbf{1 . 0 \%}(10,498)$ & $1.2 \%(69,067)$ \\
\hline Death: & $1.0 \%(5,640)$ & $1.0 \%(6756)$ & $1.0 \%(7749)$ & $1.0 \%(8983)$ & $1.0 \%(9732)$ & $0.9 \%(9664)$ & $\mathbf{0 . 9 \%}(9546)$ & $1.0 \%(58,070)$ \\
\hline $\begin{array}{l}\text { DVT/ } \\
\text { Thrombo- } \\
\text { phlebitis: }\end{array}$ & $\mathbf{0 . 6 \%}(3,315)$ & $0.6 \%(3887)$ & $0.6 \%(4336)$ & $\mathbf{0 . 6 \%}(5090)$ & $\mathbf{0 . 6 \%}(5511)$ & $0.5 \%(5626)$ & $\mathbf{0 . 5 \%}(5242)$ & $0.6 \%(33,007)$ \\
\hline Deep SSI: & $\mathbf{0 . 6 \%}(3,149)$ & $\mathbf{0 . 6 \%}(4121)$ & $0.6 \%(4846)$ & $\mathbf{0 . 6 \%}(5085)$ & $\mathbf{0 . 4 \%}(4226)$ & $\mathbf{0 . 4 \%}(3960)$ & $\mathbf{0 . 4 \%}(3779)$ & $\mathbf{0 . 5} \%(29,166)$ \\
\hline $\begin{array}{l}\text { Myocardial } \\
\text { infarction: }\end{array}$ & $\mathbf{0 . 4 \%}(1,997)$ & $\mathbf{0 . 3 \%}(2102)$ & $0.3 \%(2617)$ & $\mathbf{0 . 4 \%}(3338)$ & $\mathbf{0 . 4 \%}(3792)$ & $\mathbf{0 . 4 \%}(3853)$ & $0.4 \%(3607)$ & $\mathbf{0 . 4 \%}(21,306)$ \\
\hline $\begin{array}{l}\text { Cardiac } \\
\text { arrest: }\end{array}$ & $\mathbf{0 . 3 \%}(1,616)$ & $\mathbf{0 . 3 \%}(2003)$ & $\mathbf{0 . 3 \%}(2351)$ & $0.3 \%(2824)$ & $\mathbf{0 . 3 \%}(2957)$ & $0.3 \%(3056)$ & $\mathbf{0 . 3 \%}(2859)$ & $\mathbf{0 . 3 \%}(17,666)$ \\
\hline $\begin{array}{l}\text { Acute renal } \\
\text { fail: }\end{array}$ & $\mathbf{0 . 3} \%(1,730)$ & $\mathbf{0 . 3 \%}(1946)$ & $\mathbf{0 . 3 \%}(2178)$ & $0.3 \%(2410)$ & $\mathbf{0 . 3 \%}(2577)$ & $\mathbf{0 . 2 \%}(2469)$ & $0.2 \%(2448)$ & $\mathbf{0 . 3 \%}(15,758)$ \\
\hline $\begin{array}{l}\text { Pulmonary } \\
\text { embolism: }\end{array}$ & $\mathbf{0 . 3 \%}(1,800)$ & $\mathbf{0 . 3 \%}(2132)$ & $\mathbf{0 . 3 \%}(2514)$ & $0.3 \%(3066)$ & $\mathbf{0 . 3 \%}(3229)$ & $0.3 \%(3367)$ & $0.3 \%(3179)$ & $\mathbf{0 . 3 \%}(19,287)$ \\
\hline CVA/Stroke: & $\mathbf{0 . 2} \%(1,083)$ & $\mathbf{0 . 2 \%}(1318)$ & $\mathbf{0 . 2} \%(1414)$ & $\mathbf{0 . 2 \%}(1742)$ & $\mathbf{0 . 2 \%}(1981)$ & $0.2 \%(1970)$ & $\mathbf{0 . 2} \%(1843)$ & $\mathbf{0 . 2 \%}(11,351)$ \\
\hline $\begin{array}{l}\text { Total com- } \\
\text { plication } \\
\text { rate: }\end{array}$ & $\begin{array}{l}\mathbf{1 3 . 9 \%} \\
(75,561)\end{array}$ & $\begin{array}{l}13.8 \% \\
(89,985)\end{array}$ & $\begin{array}{l}\mathbf{1 3 . 9 \%} \\
(104,360)\end{array}$ & $\begin{array}{l}\mathbf{1 3 . 6 \%} \\
(120,140)\end{array}$ & $\begin{array}{l}\mathbf{1 3 . 0 \%} \\
(129,571)\end{array}$ & $\begin{array}{l}\mathbf{1 2 . 8 \%} \\
(131,184)\end{array}$ & $\begin{array}{l}\mathbf{1 2 . 5 \%} \\
(127,157)\end{array}$ & $13.2 \%(777,958)$ \\
\hline
\end{tabular}

Bold font indicates incidence rates for the 14 studied postoperative complications for all surgical procedures combined

Values are listed by procedure year as incidence (\%) and number of cases

OR operating room, SSI surgical site infection, DVT deep venous thrombosis, CVA cerebrovascular accident

in the U.S. could therefore exceed 30 million in total [17]. The NSQIP database includes major surgical procedures in both the inpatient and outpatient setting [18]. If the results of this study can be extrapolated to national practice in the US, a total complication incidence rate of $12.5 \%$ in 2018 (Table 2) applied to the estimated number 


\section{All surgical procedures}

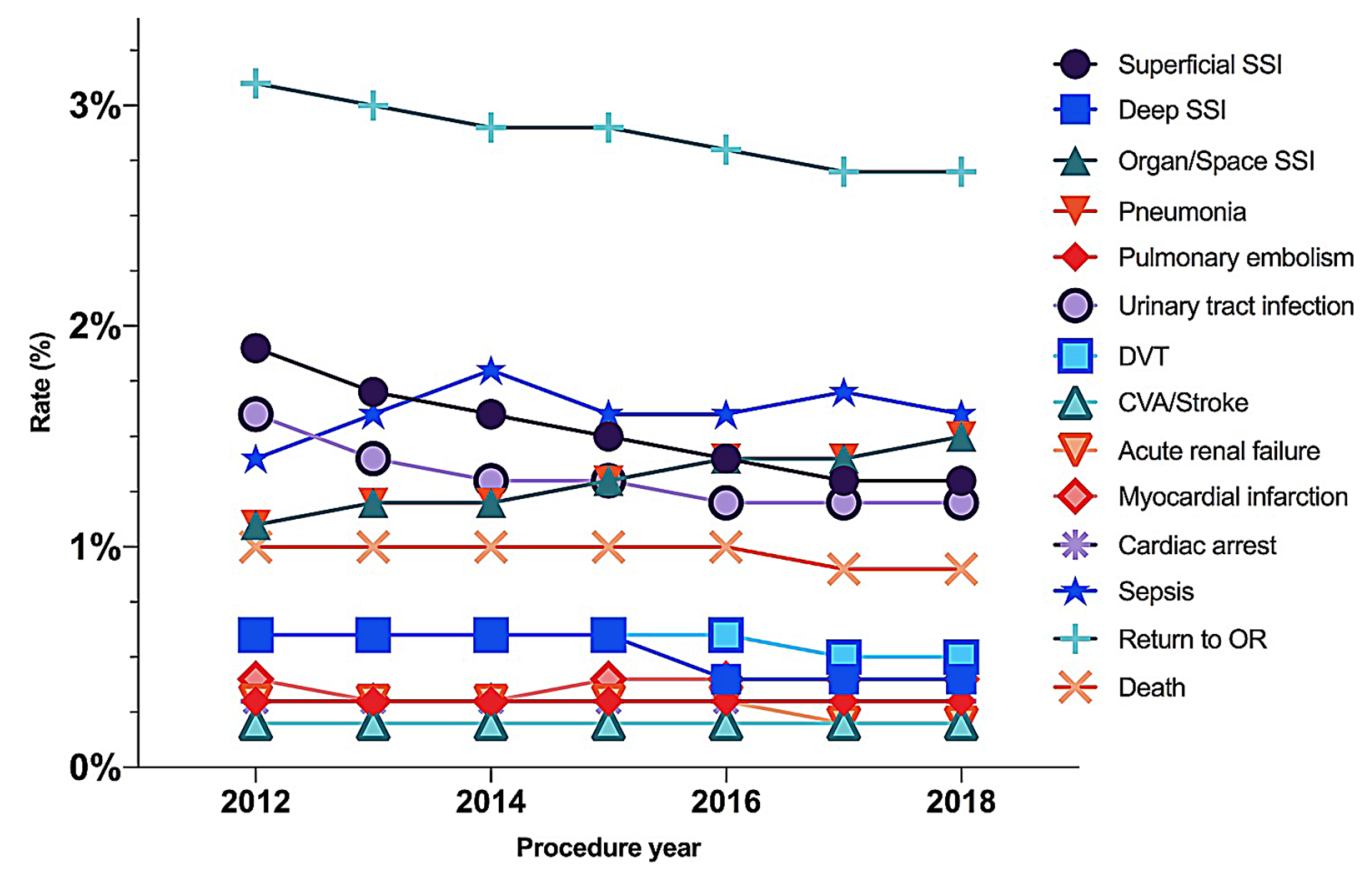

Fig. 2 Incidence rates (\%) over the study period for the 14 studied postoperative complications for all surgical procedures combined. SS/ surgical site infection; DVT deep venous thrombosis, CVA cerebrovascular accident, OR operating room

of nationwide surgical procedures would mean that $3,750,000$ postoperative complications occurred in the US in 2018. This estimate is in all probability lower than the actual number of postoperative complications as this study only includes fourteen different complications. According to Dimick et al., the mean hospital costs were estimated to be $\$ 11,626$ higher for patients with major postoperative complications, while Healy et al. estimated the additional healthcare costs caused by complications to be $\$ 19,626[7,9]$. This would correspond to the expense of \$43-73 billion in additional healthcare costs caused by postoperative complications.

Overall, the literature on postoperative complication trends across surgical subspecialities is limited. The majority of studies focus on either surgical subspecialty, type of complication or both and reported results vary. A recent NSQIP-based study shows trends of decreasing incidence rates for various complications following total knee arthroplasty with a decrease in total complication rates in the most recent cohort (2014-2016) compared to the 2006-2009 cohort (OR 0.70) [10]. Another NSQIP study examining complications following colorectal surgery also found declining rates for most complications including SSI, urinary tract infection, sepsis, DVT, reoperation and mortality [19].
On the contrary, a study of trends in surgical outcomes for rectal cancer found no significant improvement in morbidity or mortality for the two studied cohorts over a 12-year period [20]. An earlier study (2002) of 4700 inpatient procedures reported considerably higher complication rates (overall $32.1 \%$, varying from 26.9 to $42.4 \%$ ) within four subspecialties [21].

The large span within results in the literature could have several explanations-most obvious the study design. The above-mentioned studies included between 4700 and 310,000 cases, some within a highly specialized surgical field. In contrast, this study includes over 6 million patients from an array of subspecialities, which could in part account for the difference in findings. We did, however, find a statistically significant decrease of several complications, most noticeable for deep SSI, superficial SSI, acute renal failure and urinary tract infection and minor decreases for DVT and pneumonia (Table 3), which agrees fairly with some previous findings.

Part of these declining rates could be associated with increased adherence to pre-emptive strategies for prophylactic antibiotics, thrombosis prophylaxis and sepsis control management etc. [22, 23], although studies have suggested that prophylactic guidelines are often not followed when real-world data is analyzed [24]. 

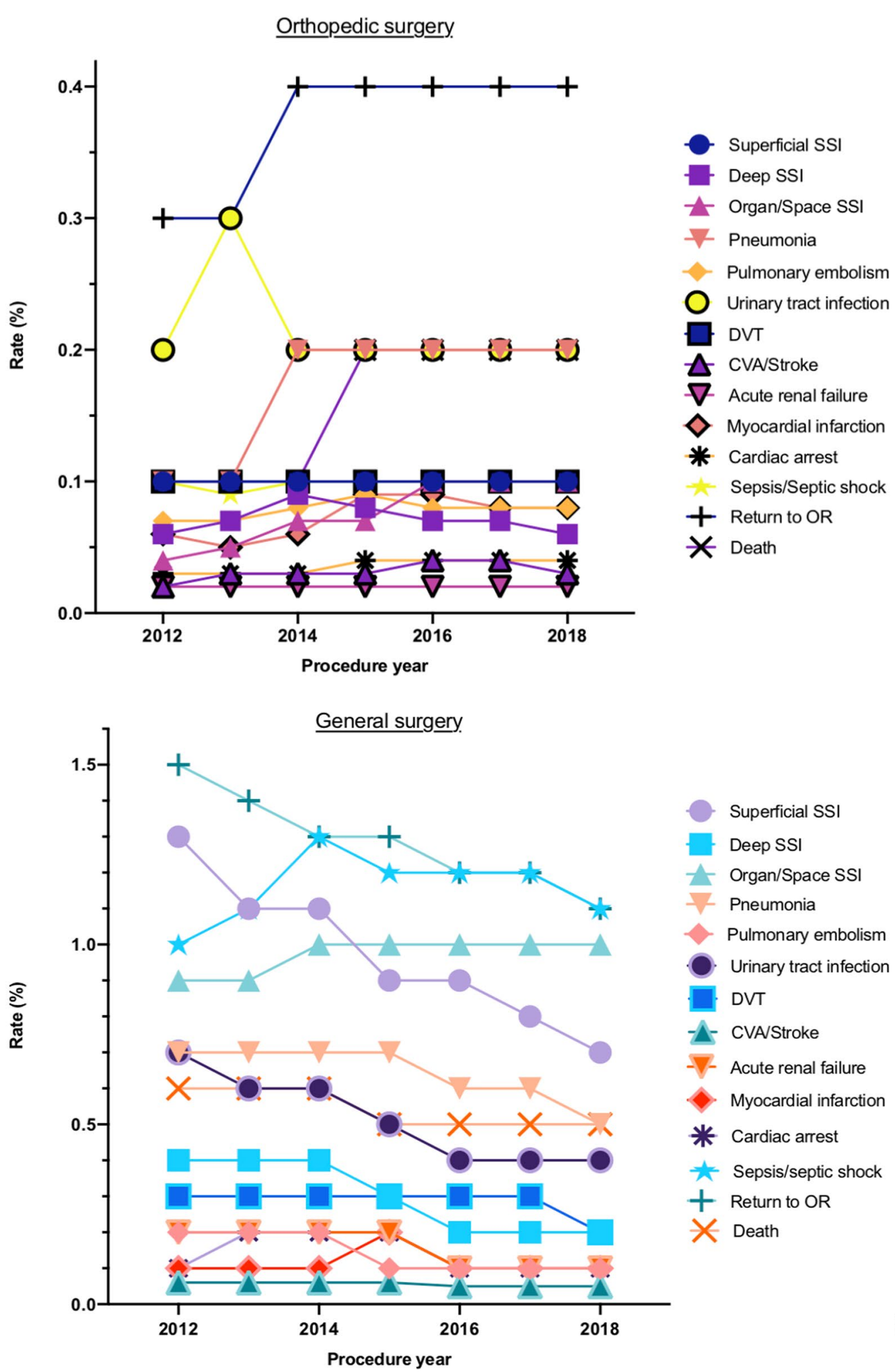

Superficial SSI

Deep SSI

Organ/Space SSI

Pneumonia

Pulmonary embolism

Urinary tract infection

DVT

CVAStroke

- - Acute renal failure

Myocardial infarction

* Cardiac arrest

* Sepsis/septic shock

+ Return to OR

X Death

Fig. 3 Postoperative complication incidence rates from 2012 to 2018 for orthopedic (top) and general (bottom) surgery. SSI: Surgical Site Infection; DVT: Deep Venous Thrombosis; CVA: Cerebrovascular Accident: OR: Operating Room 
Table 3 Results of the regression models; a univariable model and a corrected model (corrected for sex, age, Body Mass Index and American Society of Anesthesia (ASA)-classification score, diabetes, chronic obstructive pulmonary disease (COPD), blood transfusion, smoking, preoperative renal failure hypertension requiring medication and surgical subspecialty

\begin{tabular}{|c|c|c|c|c|c|c|}
\hline \multirow[t]{2}{*}{ Complications } & \multicolumn{3}{|c|}{ Corrected model } & \multicolumn{3}{|c|}{ Univariate model } \\
\hline & Odds Ratio & $95 \% \mathrm{Cl}$ & P-value & Odds Ratio & $95 \% \mathrm{Cl}$ & P-value \\
\hline Organ/space surgical site infection & 1.05 & {$[1.05-1.06]$} & $<0.01$ & 1.05 & {$[1.05-1.06]$} & $<0.01$ \\
\hline Myocardial infarction & 1.01 & {$[1.01-1.02]$} & $<0.01$ & 1.01 & {$[1.00-1.02]$} & 0.01 \\
\hline Sepsis/Septic Shock & 1.01 & {$[1.01-1.01]$} & $<0.01$ & 1.01 & {$[1.01-1.02]$} & $<0.01$ \\
\hline Pulmonary embolism & 0.99 & {$[0.98-0.99]$} & $<0.01$ & 0.99 & {$[0.98-1.00]$} & 0.01 \\
\hline Cardiac arrest requiring CPR & 0.98 & {$[0.98-0.99]$} & $<0.01$ & 0.99 & [0.98-0.99] & $<0.01$ \\
\hline CVA/Stroke w. neurological deficit & 0.99 & {$[0.98-1.00]$} & $<0.01$ & 0.99 & {$[0.98-1.00]$} & $<0.01$ \\
\hline Pneumonia & 0.98 & {$[0.97-0.98]$} & $<0.01$ & 0.97 & {$[0.97-0.98]$} & $<0.01$ \\
\hline Death & 0.98 & {$[0.97-0.98]$} & $<0.01$ & 0.98 & {$[0.97-0.98]$} & $<0,01$ \\
\hline Unplanned return to OR & 0.98 & [0.98-0.99] & $<0.01$ & 0.97 & {$[0.97-0.98]$} & $<0.01$ \\
\hline DVT/thrombophlebitis & 0.97 & {$[0.97-0.98]$} & $<0.01$ & 0.97 & {$[0.97-0.98]$} & $<0.01$ \\
\hline Urinary tract infection & 0.96 & {$[0.95-0.96]$} & $<0.01$ & 0.96 & {$[0.96-0.96]$} & $<0.01$ \\
\hline Acute renal fail & 0.96 & {$[0.95-0.96]$} & $<0.01$ & 0.95 & {$[0.94-0.96]$} & $<0.01$ \\
\hline Superficial surgical site infection & 0.94 & {$[0.94-0.94]$} & $<0.01$ & 0.94 & {$[0.94-0.94]$} & $<0.01$ \\
\hline Deep surgical site infection & 0.92 & {$[0.92-0.93]$} & $<0.01$ & 0.90 & {$[0.90-0.91]$} & $<0.01$ \\
\hline
\end{tabular}

Odds ratios (OR) and $95 \%$ confidence intervals and $\mathrm{p}$-values are listed. Odds Ratio's represent results from the logistical regression model associating the occurrence of a given complication with study year whilst correcting for confounders

$D V T$ deep venous thrombosis, CVA cerebrovascular accident, $O R$ operating room

It is, however, interesting to note that the incidence rates for organ/space SSI, MI and sepsis do not exhibit a decrease in OR over time when corrected for comorbidities.

A reason for this slight increase in OR for organ/space SSI over time could in part be due to the increasing proportion of minimal invasive procedures compared to open procedures in abdominal surgery. This would most likely reduce the number of superficial SSIs and potentially also deep SSIs, but not affect the number of organ/ space SSIs.

The observed results for sepsis mirror reports indicating that incidence rates are stable or somewhat increasing (depending on criteria/claims) from 2009 to 2014 [25]. Results regarding MI incidences seem to be conflicting with reports indicating an overall reduced incidence in the general population [26, 27]. Although this remains speculative, the presented results could indicate an increase in the diagnostic precision of MI rather than an actual increase in incidence.

Finally, it is important to note that although we observed variations in incidences of various complications, we cannot conclude on whether this is due to changes in treatment practices or changes in the underlying patient comorbidity profiles over time.

Although incidence rates of all complications exhibit significant developments over the study period (Table 3), these findings should be interpreted with caution. Due to the large patient cohort, even smaller variations will be statistically significant when performing regression analyses, without constituting an actual clinically important decline or increase. Indeed, variations in complication incidences over the study period were minor, collectively indicating that despite recent efforts in reducing complication rates, a somewhat stable subset of patients remain refractory to various prophylactic treatment efforts. This issue is again highlighted by the presented odds ratios, indicating that the overall magnitude of the effect was limited, even though findings were significant.

These patients could potentially benefit from novel precision medicine-based approaches aimed at identifying high-risk patients prior to surgery, although these approaches would require extensive validation efforts followed by large scale clinical trial efforts. To our knowledge, such trials targeting common complications in diverse surgical cohorts, have yet to be fielded.

This study provides high quality, multi-centered data and is based on a large patient cohort, yet it has its limitations. First, NSQIP complications are only registered during the 30-day follow-up period. This might result in an underestimation of the actual complication incidence rates. Secondly, NSQIP participation hospitals are often large academic centers, where complication rates could be different than non-academic facilities. This would result in data not being representative for the entire 
country and the incidence rates would in all probability be higher than reported in this study.

Third, as the study design was retrospective, we were not able to examine the cause/effect relationships and consequently this study will provide associations, but not causality.

\section{Conclusion}

In conclusion the incidence rates for the studied complications exhibit a somewhat stable trend comprising smaller changes during the study period. Future research should address how to identify patients at risk for PCspossibly by applying personalized medicine for pre- and perioperative risk assessment.

\section{Abbreviations}

NSQIP: National Surgical Quality Improvement Program; PC: Postoperative complication; SSI: Surgical site infection; DVT: Deep venous thrombosis; OR: Odds ratio; AHRQ: Agency for Healthcare Research and Quality; ACS: American College of Surgeons; SCR: Surgical risk calculator; COPD: Chronic obstructive pulmonary disease.

\section{Supplementary Information}

The online version contains supplementary material available at https://doi. org/10.1186/s12893-021-01392-z.

Additional file 1: Table S1. Missing data for variables "Sex", "Age"', Subspecialty" and the fourteen studied complications. Values are listed by procedure year as number of missing data and percentage for the year in question (\%). Table S2. Incidence rates for the 14 studied postoperative complications stratified into surgical subspecialties.

\section{Acknowledgements}

None.

\section{Authors' contributions}

$E E D, A B, K V$ and $M S$ conceived the study and performed data analysis. EED prepared the manuscript draft, which was critically revised by $A B, K V$, MS and AT. All authors read and approved the final manuscript.

\section{Funding}

The study was funded by a grant from the Novo Nordisk Foundation (Grant \#NNF19OC0055183) to MS. The funding body provided an unrestricted grant for the purpose of this research, and took no part in procurement of data, analyses, manuscript preparation or any other part of the presented work.

\section{Availability of data and materials}

Data available to participating member hospitals of the ACS NSQIP upon reasonable request.

\section{Declarations}

Ethics approval and consent to participate

The study was approved by the American College of Surgeons and the Massachusetts General Hospital Institutional Review Board (protocol approval \#2021P001182).

\section{Consent for publication}

Not applicable. All patient data is de-identified and assembled in in a quality control database through the HIPAA compliant ACS NSQIP collaborative.
Patient consent for NSQIP participation is provided in writing as per local NSQIP participating hospital protocols.

\section{Competing interests}

The authors declare that they have no competing interests.

\section{Author details}

${ }^{1}$ Department of Surgical Gastroenterology and Transplantation C-TX, Copenhagen University Hospital, Blegdamsvej 9, Copenhagen Ø, 2100 Rigshospitalet, Denmark. ${ }^{2}$ Center for Surgical Translational and Artificial Intelligence Research (CSTAR), Copenhagen University Hospital, Rigshospitalet, Denmark. ${ }^{3}$ Department of Orthopedics, Copenhagen University Hospital, Hvidovre, Denmark. ${ }^{4}$ Harris Orthopedics Laboratory, Massachusetts General Hospital, Boston, USA. ${ }^{5}$ Institute of Clinical Medicine, University of Copenhagen, Hvidovre, Denmark.

Received: 17 June 2021 Accepted: 28 October 2021

Published online: 06 November 2021

\section{References}

1. Weiser TG, Haynes AB, Molina G, Lipsitz SR, Esquivel MM, Uribe-LeitzT, et al. Size and distribution of the global volume of surgery in 2012. Bull World Health Organ. 2016;94(3):201-9.

2. Tevis SE, Cobian AG, Truong HP, Craven MW, Kennedy GD. Implications of multiple complications on the postoperative recovery of general surgery patients. Ann Surg. 2016;263(6):1213-8.

3. Haynes AB, Weiser TG, Berry WR, Lipsitz SR, Breizat AH, Dellinger EP, et al. A surgical safety checklist to reduce morbidity and mortality in a global population. N Engl J Med. 2009;360(5):491-9.

4. Tevis SE, Kennedy GD. Postoperative complications and implications on patient-centered outcomes. J Surg Res. 2013;181(1):106-13.

5. Straatman J, Cuesta MA, de Lange-de Klerk ES, van der Peet DL. Hospital cost-analysis of complications after major abdominal surgery. Dig Surg. 2015;32(2):150-6.

6. Clavien PA, Sanabria JR, Strasberg SM. Proposed classification of complications of surgery with examples of utility in cholecystectomy. Surgery. 1992;111(5):518-26

7. Healy MA, Mullard AJ, Campbell DA Jr, Dimick JB. Hospital and payer costs associated with surgical complications. JAMA Surg. 2016;151(9):823-30.

8. Vonlanthen R, Slankamenac K, Breitenstein S, Puhan MA, Muller MK, Hahnloser D, et al. The impact of complications on costs of major surgical procedures: a cost analysis of 1200 patients. Ann Surg. 2011;254(6):907-13

9. Dimick JB, Chen SL, Taheri PA, Henderson WG, Khuri SF, Campbell DA Jr. Hospital costs associated with surgical complications: a report from the private-sector National Surgical Quality Improvement Program. J Am Coll Surg. 2004;199(4):531-7.

10. Sarpong NO, Boddapati V, Herndon CL, Shah RP, Cooper HJ, Geller JA. Trends in length of stay and 30-day complications after total knee arthroplasty: an analysis from 2006 to 2016. J Arthroplasty. 2019;34(8):1575-80.

11. American College of Surgeons. ACS national surgical quality improvement program. https://www.facs.org/quality-programs/acs-nsqip; 2018

12. American College of Surgery. Userguide for the ACS NSQIP participant use data file. 2018. https://www.facs.org/quality-programs/acs-nsqip/ participant-use.

13. Stavseth MR, Clausen T, Røislien J. The clinical consequences of variable selection in multiple regression models: a case study of the Norwegian Opioid Maintenance Treatment program. Am J Drug Alcohol Abuse. 2020;46(1):13-21.

14. R Core Team (2017). R: A language and environment for statistical computing. R Foundation for Statistical Computing, Vienna, Austria. URL https://www.R-project.org/.

15. Agency for Healthcare Reasearch and Quality (AHRQ). Healtcare Cost and Utilization Project. 2017. Statistical Brief \#233. https://www.hcup-us. ahrq.gov/reports/statbriefs/sb233-Operating-Room-Procedures-UnitedStates-2014.pdf.

16. Agency for Healthcare Reasearch and Quality (AHRQ). Healtcare Cost and Utilization Project. 2015. Statistical Brief \#188. https://www.hcup-us.ahrq. gov/reports/statbriefs/sb188-Surgeries-Hospital-Outpatient-Facilities2012.pdf 
17. Agency for Healthcare Reasearch and Quality (AHRQ). Healtcare Cost and Utilization Project. 2017. Statistical Brief \#233. https://www.hcup-us. ahrq.gov/reports/statbriefs/sb233-Operating-Room-Procedures-UnitedStates-2014.pdf.

18. American College of Surgery. Userguide for the ACS NSQIP participant use data file. 2018. https://www.facs.org/quality-programs/acs-nsqip/ participant-use.

19. Al-Mazrou AM, Haiqing Z, Guanying Y, Kiran RP. Sustained positive impact of ACS-NSQIP program on outcomes after colorectal surgery over the last decade. Am J Surg. 2020;219(1):197-205.

20. Sharp SP, Malizia R, Skancke M, Arsoniadis EG, Ata A, Stain SC, et al. A NSQIP analysis of trends in surgical outcomes for rectal cancer: What can we improve upon? Am J Surg. 2020;220(2):401-7.

21. Healey MA, Shackford SR, Osler TM, Rogers FB, Burns E. Complications in surgical patients. Arch Surg. 2002;137(5):611-7.

22. van Zanten AR, Brinkman S, Arbous MS, Abu-Hanna A, Levy MM, de Keizer NF. Guideline bundles adherence and mortality in severe sepsis and septic shock. Crit Care Med. 2014;42(8):1890-8.

23. Segala FV, Murri R, Taddei E, Giovannenze F, Del Vecchio P, Birocchi E, et al. Antibiotic appropriateness and adherence to local guidelines in perioperative prophylaxis: results from an antimicrobial stewardship intervention. Antimicrob Resist Infect Control. 2020;9(1):164.

24. Randelli F, Cimminiello C, Capozzi M, Bosco M, Cerulli G. Real life thromboprophylaxis in orthopedic surgery in Italy. Results of the GIOTTO study. Thromb Res. 2016;137:103-7.

25. Rhee C, Dantes R, Epstein L, Murphy DJ, Seymour CW, Iwashyna TJ, et al. Incidence and trends of sepsis in US hospitals using clinical vs claims data, 2009-2014. JAMA. 2017;318(13):1241-9.

26. Hariri E, Tisminetzky M, Lessard D, Yarzebski J, Gore J, Goldberg R. Twenty-five-year (1986-2011) trends in the incidence and death rates of stroke complicating acute myocardial infarction. Am J Med. 2018;131(9):1086-94.

27. Yeh RW, Sidney S, Chandra M, Sorel M, Selby JV, Go AS. Population trends in the incidence and outcomes of acute myocardial infarction. N Engl J Med. 2010;362(23):2155-65

\section{Publisher's Note}

Springer Nature remains neutral with regard to jurisdictional claims in published maps and institutional affiliations.
Ready to submit your research? Choose BMC and benefit from:

- fast, convenient online submission

- thorough peer review by experienced researchers in your field

- rapid publication on acceptance

- support for research data, including large and complex data types

- gold Open Access which fosters wider collaboration and increased citations

- maximum visibility for your research: over $100 \mathrm{M}$ website views per year

At BMC, research is always in progress.

Learn more biomedcentral.com/submissions 